\title{
Origin of metallic surface core-level shifts
}

\author{
Aldén, Magnus; Skriver, Hans Lomholt; Abrikosov, I. A.; Johansson, Börje
}

Published in:

Physical Review B

Link to article, DOI:

10.1103/PhysRevB.51.1981

Publication date:

1995

Document Version

Publisher's PDF, also known as Version of record

Link back to DTU Orbit

Citation (APA):

Aldén, M., Skriver, H. L., Abrikosov, I. A., \& Johansson, B. (1995). Origin of metallic surface core-level shifts. Physical Review B, 51(3), 1981-1984. https://doi.org/10.1103/PhysRevB.51.1981

\section{General rights}

Copyright and moral rights for the publications made accessible in the public portal are retained by the authors and/or other copyright owners and it is a condition of accessing publications that users recognise and abide by the legal requirements associated with these rights.

- Users may download and print one copy of any publication from the public portal for the purpose of private study or research.

- You may not further distribute the material or use it for any profit-making activity or commercial gain

- You may freely distribute the URL identifying the publication in the public portal

If you believe that this document breaches copyright please contact us providing details, and we will remove access to the work immediately and investigate your claim 


\title{
Origin of metallic surface core-level shifts
}

\author{
Magnus Aldén \\ Cap Programator, Box 825, S-16124 Bromma, Sweden \\ H. L. Skriver \\ Center for Atomic-Scale Materials Physics and Physics Department, Technical University of Denmark, \\ DK-2800 Lyngby, Denmark \\ I. A. Abrikosov and Börje Johansson \\ Condensed Matter Theory Group, Physics Department, Uppsala University, S-75121 Uppsala, Sweden
}

(Received 7 July 1994)

\begin{abstract}
The unique property of the open $4 f$ energy shell in the lanthanide metals is used to show that the initial-state energy shift gives an insufficient description of surface core-level shifts. Instead, a treatment, that fully includes the final-state screening, accounts for the experimentally observed surface shifts of the occupied as well as the unoccupied $4 f$ states. The surface energy shift of the initial state corresponds approximately to the average of the shifts for the occupied and unoccupied $4 f$ levels.
\end{abstract}

Surface core-level energy shifts (SCS) for elemental metals have been known for about one and a half decades. ${ }^{1}$ During this time the origin of SCS has been under a considerable debate ${ }^{2-7}$ where especially the importance of final-state effects has been in focus. ${ }^{6}$ In many theoretical treatments it is assumed that the final-state screening effects are the same at the surface and in the bulk. Accordingly, it has been argued that the physical origin of the shift is to be found in the initial-state shift of the core-level binding energy., ${ }^{3,4}$ Other theories maintain that in addition to this the final state is also subject to a surface shift, which has to be incorporated into the theory before a full account of the measured surface shift can be given. ${ }^{2}$ In the present work, a unique property of the open $4 f$ shell in the lanthanide elements is used to resolve this longstanding controversy.

Due to advances in the preparation techniques for single-crystal surfaces of the lanthanide metals as well as improved resolution and surface sensitivity in inverse photoemission experiments, much improved experimental data have recently become available both for several occupied as well as for a few unoccupied $4 f$ states. ${ }^{8,9} \mathrm{Si}-$ multaneously, a corresponding improvement in the theoretical description has taken place, permitting an accurate study of surface core-level shifts. ${ }^{10-12}$ The lanthanides are characterized by an open $4 f$ shell, with an integral occupation number $n$, which, as far as bonding is concerned, ${ }^{13}$ is inert and therefore is completely equivalent to a genuine core level. They therefore constitute an ideal set of materials where the origin of the SCS may be clarified.

Here we focus on the fact that there will be a surface shift of the $4 f$ removal as well as the $4 f$ addition energy, i.e., both the x-ray photoelectron (XPS) spectrum and the bremsstrahlung isochromat (BIS) spectrum will contain energy shifted $4 f$ signals. The extraordinary circumstance which allows us to address the question of the origin of the SCS is now that the initial-state shift of the $4 f$ level is identical in both the electron removal and the electron addition process. If the initial-state picture of the SCS were correct, it would immediately follow that the surface energy shifts of the $4 f^{n} \rightarrow 4 f^{n-1}$ and $4 f^{n} \rightarrow 4 f^{n+1}$ processes should be identical. In this paper we show that this is not the case and that the initial-state shift does not give an adequate description of the SCS. Recent experimental data give full support to this conclusion.

The $a b$ initio calculations of the surface shifts of the occupied and unoccupied $4 f$ levels for the lanthanide metals which we present include the initial- and final-state effects within the assumption of a complete screening for the $4 f$-removal and $4 f$-addition processes, i.e., the main line of the spectral features corresponds to an electronically completely relaxed final state. ${ }^{14,15}$ Furthermore, for the treatment of the screened $4 f$-addition state, the $4 f^{n+1}$ configuration is assumed to be localized, without any significant hybridization with the valence states, ${ }^{15}$ and similarly for the screened $4 f$-removal state.

The complete screening picture implies that the surface shift of the occupied and unoccupied $4 f$ levels becomes equal to the surface segregation energy ${ }^{2}$ of a substitutional $4 f^{n-1}$ or $4 f^{n+1}$ impurity, respectively, in the $4 f^{n}$ metal host. Since most of the lanthanides are trivalent in the metallic state, this corresponds to the surface segregation of a tetravalent or divalent impurity, respectively, in the trivalent host. Due to the vertical nature of the electronic transitions, the impurities in question are not associated with lattice relaxations of the surrounding host atoms, a most attractive feature for the theoretical treatment.

We use a Green's function technique within the scalâr-relativistic tight-binding linear muffin-tin orbitals method as implemented for surfaces and interfaces by Skriver and Rosengaard, ${ }^{16}$ employing the frozen-core and the atomic-sphere approximations. The impurity formulation is essentially that given previously by Gun- 
narsson, Jepsen, and Andersen, ${ }^{17}$ but here used within the most localized (tight-binding) representation ${ }^{18}$ and implemented separately, at the same level of approximation, for the bulk and surface environments. ${ }^{12}$ The one-electron potential is computed within local-density theory ${ }^{19}$ and the atomic-sphere approximation, including both monopole and dipole contributions to the intersphere electrostatic potential. The $4 f$ electrons are included in the frozen-core approximation, whereby magnetic effects are not accounted for. Finally, surface layer relaxations are neglected. Relevant to the present study is the previous investigations by means of the present Green's function technique of the surface energy ${ }^{16}$ and surface core-level shift ${ }^{11,12}$ for a wide range of elements, where good agreement with experiment is obtained. In the present total energy calculations, however, we extend the atomic-sphere approximation by including the full, nonspherical charge density, whereby a previous deficiency in the surface energy for early transition metals is removed. ${ }^{20}$

The impurity which enters the separate Green's function impurity calculations in the bulk and at the surface is a neutral $4 f$-excited atom, where an electron is removed from the $4 f$ shell and placed into the valence band in the case of $4 f$ removal [denoted by $(n-1)$ ] and from the valence band into the $4 f$ shell in the case of $4 f$ addition [denoted by $(n+1)]$. The surface shift of the $4 f$ level in question may be obtained as the surface segregation energy, ${ }^{2}$

$$
\Delta_{c}^{4 f^{n \mp 1}}=E_{\mathrm{imp} ; 4 f^{n \mp 1}}^{\text {surface }}-E_{\mathrm{imp} ; 4 f^{n \mp 1}}^{\mathrm{bulk}}
$$

where $E_{\mathrm{imp} ; 4 f^{n \mp 1}}^{\text {surface }}$ and $E_{\mathrm{imp} ; 4 f^{n \mp 1}}^{\mathrm{bulk}}$ are the solution energies for the $4 f^{n \mp 1}$ impurity located in the bulk and at the surface, respectively. ${ }^{12}$ With the sign convention used in Eq. (1), a positive shift corresponds to a higher binding energy for the surface $4 f$ level than for the bulk $4 f$ level in the case of $4 f$ removal, and in the case of $4 f$ addition a positive shift corresponds to a higher energy position above the Fermi level for the surface $4 f$ level than for the bulk $4 f$ level.

The calculated surface $4 f$ level shifts for the closepacked hcp (0001) facet ${ }^{22}$ of the trivalent lanthanide metals are presented in Fig. 1 where we have changed the sign of the shift of the unoccupied levels (top thick curve), in contrast to the definition used in Eq. (1). With this sign convention the occupied and unoccupied levels move in the same direction and can be directly compared with each other. Thereby it becomes apparent that for all the elements the magnitude of the shift of the unoccupied $4 f$ states is significantly larger $(\sim 0.2 \mathrm{eV})$ than that of the occupied states. This difference in magnitude must originate from a final-state effect. Another salient feature in Fig. 1 is the gradual increase in magnitude of the shift of both the occupied and the unoccupied levels as one proceeds through the series. Since this behavior occurs in common, it may, accordingly, be regarded as an initial state effect.

To analyze the origin of the calculated behavior, we consider a simple model of the valence-electron bonding in the final state. The screening in the final state will con-

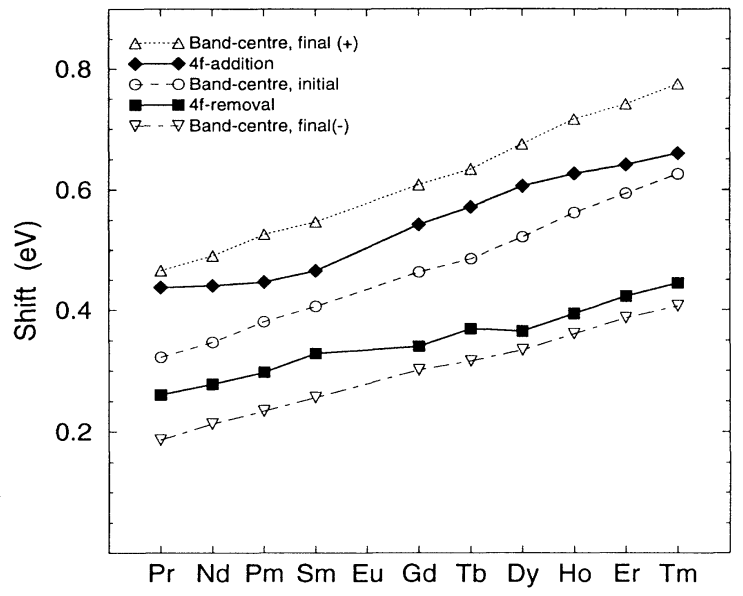

FIG. 1. Comparison between, on the one hand, $4 f$-addition surface shifts (solid diamonds) and $4 f$-removal surface shifts (solid squares) and, on the other hand, surface shift of the $d$-state band center referring to the initial state (open circles) and the two different final states of $4 f$ addition (open triangles, top curve) and $4 f$ removal (open triangles, bottom curve), respectively.

sist of essentially plus or minus one $d$ electron, depending on whether we consider the $4 f$-removal or $4 f$-addition process, respectively. ${ }^{23}$ The difference in bonding energy betwen the initial- and final states may be described by the one-electron contribution

$$
\begin{aligned}
\Delta E_{\mathrm{screen}}^{\mathrm{one-el}}= & \int^{\epsilon_{F}}\left(\varepsilon-C_{d}^{n_{d} \pm 1}\right) D_{d}^{n_{d} \pm 1}(\varepsilon) d \varepsilon \\
& -\int^{\epsilon_{F}}\left(\varepsilon-C_{d}^{n_{d}}\right) D_{d}^{n_{d}}(\varepsilon) d \varepsilon
\end{aligned}
$$

where $\epsilon_{F}$ is the Fermi level, $n_{d}$ is the number of $d$ electrons in the initial state, and $D_{d}$ and $C_{d}$ are the local $n_{d^{-}}$ dependent state density and band centroid, respectively, of the $d$ electrons. We apply a rigid band picture in which the valence states in the final state have been pulled down, corresponding to a situation with $\left(n_{d}+1\right) d$ electrons, or up [corresponding to $\left(n_{d}-1\right) d$ electrons] by the energy $\delta E$, i.e., depending on whether we consider screening due to $4 f$ removal or $4 f$ addition, respectively, so that $D_{d}^{n_{d} \pm 1}(\varepsilon)=D_{d}^{n_{d}}(\varepsilon \pm \delta E)$. For the band-center parameter it becomes convenient to introduce the transition-state centroid $C_{d}^{n_{d} \pm 1 / 2}$ through $C_{d}^{n_{d} \pm 1}=C_{d}^{n \pm 1 / 2} \mp \delta E / 2$ and $C_{d}^{n_{d}}=C_{d}^{n \pm 1 / 2} \pm \delta E / 2$. Then (2) becomes

$$
\begin{aligned}
\Delta E_{\text {screen }}^{\text {one-el }} \approx & \int^{\epsilon_{F} \pm \delta E}[\varepsilon \mp \delta E \\
& \left.-\left(C_{d}^{n_{d} \pm 1 / 2} \mp \delta E / 2\right)\right] D_{d}^{n_{d}}(\varepsilon) d \varepsilon \\
& -\int^{\epsilon_{F}}\left[\varepsilon-\left(C_{d}^{n_{d} \pm 1 / 2} \pm \delta E / 2\right)\right] D_{d}^{n_{d}}(\varepsilon) d \varepsilon
\end{aligned}
$$

which further simplifies into

$$
\Delta E_{\text {screen }}^{\text {one-el }} \approx \pm\left(\epsilon_{F}-C_{d}^{n_{d} \pm 1 / 2}\right) .
$$

The above one-electron bonding analysis may equally 
well be applied to the case of surface $(S)$ or bulk $(B)$ screening. Hence the SCS can be derived from the simple estimate

$$
\Delta_{c}^{4 f^{n \mp 1}} \approx \mp\left(C_{d}^{S ; n_{d} \pm 1 / 2}-C_{d}^{B ; n_{d} \pm 1 / 2}\right)
$$

for which the minus in front of the parentheses refers to the case of the $4 f$-removal shift and the plus refers to the $4 f$-addition shift, in accordance with the sign convention used in Eq. (1). In Fig. 1, however, we display with thin lines the computed shift of the initial $\left(C_{d}^{n_{d}}\right)$ and final $\left(C_{d}^{n_{d} \pm 1}\right)$ states, thus decomposing the transition-state $\left(C_{d}^{n_{d} \pm 1 / 2}\right)$ shift in terms of the separate effects of the initial and final states. This decomposition becomes rather natural in the rigid band approach, where the hypothetical transition state is the average of the initial and final states. Furthermore, for the purpose of presentation in Fig. 1 we consider the absolute values, i.e., $-\left(C_{d}^{S ; n_{d}}-C_{d}^{B ; n_{d}}\right)$ and $-\left(C_{d}^{S ; n_{d} \pm 1}-C_{d}^{B ; n_{d} \pm 1}\right)$, whereby the shifts become directly comparable. As can be seen the $4 f$ energy shifts from the full calculations are rather well approximated by the transition-state shift in Eq. (5), since they fall between the initial-state and the corresponding final-state band-center shifts.

The surface shift of the $d$ band center is of course electrostatically induced due to the shift in the local surface potential. The potential shift relates to the well-known situation for the transition metals, where the $d$-band narrowing in conjunction with $d$-charge conservation at the surface produces a surface core-level shift which scales with the $d$ band filling, and which changes sign in the middle of a transition series. ${ }^{3,4}$ For the present elements the initial-state surface shift of the potential (band center) is negative, in accordance with the fact that these systems are early transition metals. For the lanthanides, when one proceeds from the light to the heavier elements, the $d$ occupation is reduced from 2.0 to 1.4 electrons, and at the same time there is a gradual decrease of the atomic volume. ${ }^{21}$ As a result, there is a gradual increase in the charge transfer at the surface when proceeding from La to $\mathrm{Lu}$, which explains the enhancement of the band-center shifts with increasing atomic number as shown by the thin curves in Fig. 1.

The band-narrowing effect of the $d$ band has for transition metals commonly been used to explain the shift of the core-electron eigenvalues, in the association, or even identification, with a so-called initial-state shift. ${ }^{3,4}$ The surface potential is simultaneously responsible for the shift of the core eigenvalue and of the valence band center. We prefer to interpret the initial-state effect by the notion that at the surface the screening takes place in valence levels that are well shifted already in the initial state. For the lanthanides this shift increases with increasing atomic number. The shift of the band center in the final state, however, is significantly larger for the $4 f$-addition state than for the $4 f$-removal state, since its final state has almost $2 d$ electrons less than the final state for the $4 f$-removal state. ${ }^{23}$ This immediately explains the larger magnitudes for the shift of the unoccupied $4 f$ level than for the occupied $4 f$ level seen in Fig. 1.

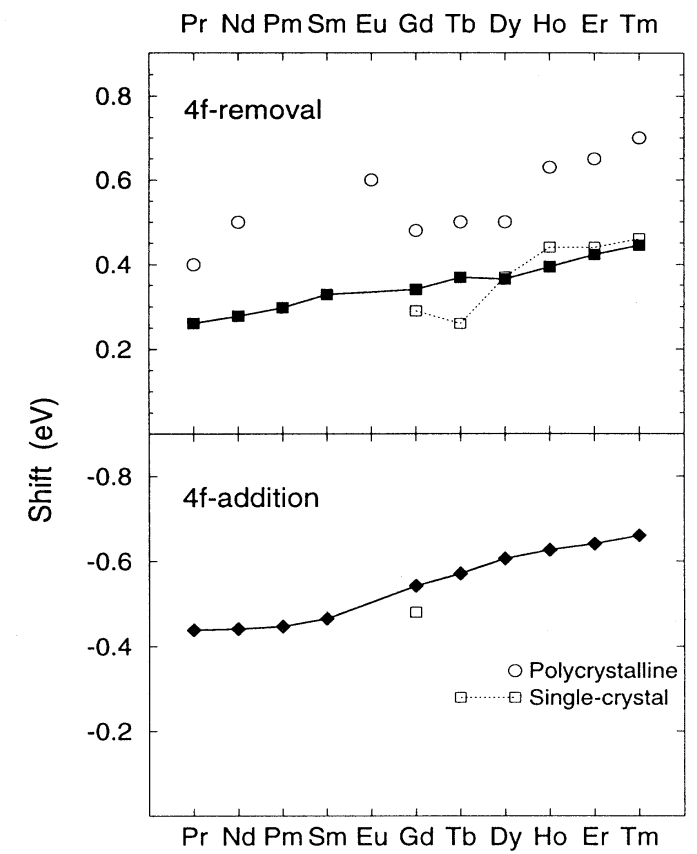

FIG. 2. Comparison between calculated and experimental values, for the surface shift of the occupied $4 f$ levels (upper, positive data), and for the surface shift of the unoccupied $4 f$ levels (lower, negative data). Calculated shifts are marked with filled symbols and thick lines. Polycrystalline and single-crystal measurements are marked with open circles and open squares, respectively.

A comparison between the theoretical data and experimental recordings of $4 f$ level shifts is presented in Fig. 2. Here we divide the experimental data into two categories, namely measurements referring to polycrystalline samples $^{7}$ (circles) and the most recent single-crystal film recordings $^{8,9}$ (squares). For the $4 f$-removal shift in the upper part of Fig. 2, it is seen that the polycrystalline measurements ${ }^{7}$ exhibit shifts which are systematically larger than our presently calculated values. The general trend across the series and the amount of increase from $\mathrm{La}$ to $\mathrm{Lu}$ is, however, similar in these two sets of data. In a previous hcp (0001) slab calculation which was made purely within the initial-state model, the shifts were computed to be significantly larger than the present ones. ${ }^{24}$ As a result (see below), a somewhat fortuitous agreement with the polycrystalline measurements was obtained in that work. ${ }^{24}$

When comparing our calculated $4 f$-removal shifts with the new high-resolution, single-crystal measurements, ${ }^{8,9}$ it is evident in Fig. 2 that the agreement is significantly improved. For Gd (Ref. 9) and Tb (Ref. 8), the experimental results are even slightly lower than our computed values, and rather surprisingly the experimental shift decreases when proceeding from $\mathrm{Gd}$ to $\mathrm{Tb}$, hence opposing the behavior seen in the older polycrystalline data ${ }^{7}$ and in the present calculations. In the most recent experimental deduction of the $4 f$-removal shift for Dy, Ho, Er, and $\mathrm{Tm},{ }^{9}$ the picture of an increase across the series appears to be valid again and the agreement between experiment 
and our present results becomes almost perfect.

For the case of the $4 f$-addition shift, the presently available experimental shifts are as yet quite limited. Only for single-crystal $\mathrm{Gd}(0001)$ has there been a simultaneous recording of the $4 f$-addition and $4 f$-removal surface shifts. ${ }^{9}$ The observed shifts were resolved to be $\mathbf{0 . 2 9}$ $\pm 0.03 \mathrm{eV}$ for the occupied $4 f$ level and $-0.48 \pm 0.04$ $\mathrm{eV}$ for the unoccupied $4 f$ level, also displayed in Fig. 2 by squares in the top and bottom panel, respectively. The magnitude of the measured $4 f$-addition shift thus exceeds that of the $4 f$-removal shift by $0.19 \pm 0.05 \mathrm{eV}$. Our presently calculated corresponding values are 0.34 $\mathrm{eV}$ and $-0.54 \mathrm{eV}$ giving a difference in magnitude of 0.20 $\mathrm{eV}$, in very good agreement with the experimental data.

In summary, we have utilized the unique properties of the $4 f$ electrons in the lanthanide metals to investigate initial- and final-state effects. The surface energy shift of the occupied and unoccupied $4 f$ levels have been computed within the concept of complete screening, i.e., through separate $4 f^{n \pm 1}$ impurity calculations in the bulk and at the surface. Thereby, a systematic $\sim 0.2 \mathrm{eV}$ differ- ence in the shifts of the occupied and unoccupied levels is obtained in quantitative agreement with recent experimental data which proves the significance of the final state. The different roles played by the initial and final states are clarified within a model of the screening properties of the $d$ electrons. It is shown that the surface shifts of the initial state approximately corresponds to the average of the occupied and unoccupied $4 f$ level shifts. The present results have important consequences for chemical shifts in general, since they clearly demonstrate the limitation of the initial state picture.

We gratefully acknowledge Professor C. Laubschat and his co-workers in Berlin for letting us include their data prior to publication. B.J., M.A., and I.A.A. are grateful for financial support from the Swedish Natural Science Research Council. The Center for Atomic-scale Materials Physics is sponsored by the Danish National Research Foundation. Part of this work was supported by grants from the Danish research councils through the Danish Center for Surface Reactivity.
${ }^{1}$ P.H. Citrin, G.K. Wertheim, and Y. Baer, Phys. Rev. Lett. 41, 1425 (1978).

2 B. Johansson and N. Mårtensson, Phys. Rev. B 21, 4427 (1980); Helv. Phys. Acta 56, 405 (1983); A. Rosengren and B. Johansson, Phys. Rev. B 23, 3852 (1981).

${ }^{3}$ P.H. Citrin and G.K. Wertheim, Phys. Rev. B 27, 3176 (1983).

${ }^{4}$ D. Spanjaard, C. Guillot, M.C. Desjonquères, G. Tréglia, and J. Lecante, Surf. Sci. Rep. 5, 1 (1985).

${ }^{5}$ W.F. Egelhoff, Surf. Sci. Rep. 6, 253 (1987).

${ }^{6}$ P.S. Bagus, G. Pacchioni, and F. Parmigiani, Phys. Rev. B 43, 5172 (1991).

${ }^{7}$ For a recent review, see A. Flodström, R. Nyholm, and B. Johansson, in Advances in Surface and Interface Science, Volume 1: Techniques, edited by R.Z. Bachrach (Plenum Press, New York, 1992).

${ }^{8}$ E. Navas, K. Starke, C. Laubschat, E. Weschke, G. Kaindl, Phys. Rev. B 48, 14753 (1993).

${ }^{9}$ A.V. Fedorov, E. Arenholz, K. Starke, E. Navas, L. Baumgarten, C. Laubschat, and G. Kaindl, Phys. Rev. Lett. 73, 601 (1994); A. Höhr, E. Weschke, S. Vaudré, C. Laubschat, and G. Kaindl (unpublished); private communication.

${ }^{10}$ P.J. Feibelman, Phys. Rev. B 39, 4866 (1989).

${ }^{11}$ M. Aldén, H.L. Skriver, and B. Johansson, Phys. Rev. Lett. 71, 2449 (1993); 71, 2457 (1993).

${ }^{12}$ M. Aldén, I.A. Abrikosov, B. Johansson, N.M. Rosengaard, and H.L. Skriver, Phys. Rev. B 50, 5131 (1994).

${ }^{13}$ B. Johansson and P. Munck, J. Less-Common Met. 100, 49 (1984).

14 J.F. Herbst, D.N. Lowy, and R.E. Watson, Phys. Rev. B 6, 1913 (1972); J.F. Herbst, R.E. Watson, J.W. Wilkins, ibid. 17, 3089 (1978).
${ }^{15}$ B. Johansson, Phys. Rev. B 20, 1315 (1979).

${ }^{16}$ H.L. Skriver and N.M. Rosengaard, Phys. Rev. B 43, 9538 (1991); 46, 7157 (1992).

${ }^{17}$ O. Gunnarsson, O. Jepsen, and O.K. Andersen, Phys. Rev. B 27, 7144 (1983).

${ }^{18}$ O.K. Andersen and O. Jepsen, Phys. Rev. Lett. 53, 2571 (1984); O.K. Andersen, Z. Pawlowska, and O. Jepsen, Phys. Rev. B 34, 5253 (1986).

${ }^{19}$ S.H. Vosko, L. Wilk, and M. Nusair, Can. J. Phys. 58, 1200 (1980).

${ }^{20}$ L. Vitos, J. Kollár, and H.L. Skriver, Phys. Rev. B 49, 16 694 (1994); J. Kollár, L. Vitos, and H.L. Skriver, ibid. 49, 11288 (1994).

${ }^{21}$ H.L. Skriver, in Systematics and the Properties of the Lanthanides, edited by S.P. Sinha (Reidel, Dordrecht, 1983), p. 239.

${ }^{22}$ We consistently use the hcp structure for all the investigated elements, thus approximating the Sm phase and the earlier lanthanides forming in the dhcp structure. Similarly, in the present work we only treat trivalent lanthanides. Hence, the abnormal transition to a divalent surface for $\mathrm{Sm}$ is not considered.

${ }^{23}$ From explicit calculations we have found that the screening charge is close to one $5 d$ electron upon $4 f$ removal, whereas for the $4 f$ addition the lost screening charge consists of $0.85 d$ electrons, and the rest is of $6 s 6 p$ character. For our simplified analysis we let the screening charge be $n_{d}+1$ $\left(n_{d}-1\right)$ for the $4 f$ removal (addition) process, where $n_{d}$ is the $d$-occupation number for the $4 f^{n}$ metal.

${ }^{24}$ A.M. Begley, R.G. Jordan, W.M. Temmerman, and P.I. Durham, Phys. Rev. B 41, 11780 (1990). 